\title{
Enhancing biomass and lipid accumulation in the microalgae Schizochytrium sp. by addition of fulvic acid and EDTA
}

\author{
Xiao-Man Sun ${ }^{1}$, Lu-Jing Ren ${ }^{1,4^{*}}$, Xiao-Jun Ji ${ }^{1,4}$ and He Huang ${ }^{2,3,4}$ (D)
}

\begin{abstract}
Enhancing lipid productivity and reducing oxidative damage is essential for lipid overproduction in microalgae. In this study, addition of $20 \mathrm{mg} / \mathrm{L}$ fulvic acid (FA) resulted a 34.4\% increase of lipid yield in Schizochytrium sp. Furthermore, the cooperative effect of FA and EDTA on cell growth and lipid production was investigated. The combined addition of $20 \mathrm{mg} / \mathrm{L}$ FA and $1.0 \mathrm{~g} / \mathrm{L}$ EDTA yielded a maximal cell dry weight of $130.7 \mathrm{~g} / \mathrm{L}$ and lipid productivity of $1.16 \mathrm{~g} / \mathrm{L} / \mathrm{h}$, representing $36.4 \%$ and threefold increase over the non-supplemented group, respectively. Moreover, compared with the non-supplemented group, the combined addition strategy exhibited overall lower levels of reactive oxygen species and malondialdehyde, which accompanied with $66.7 \%$ and $81.9 \%$ higher superoxide dismutase and catalase activity, respectively. Furthermore, a $24.1-37.1 \%$ increase of malic enzyme and 19.4-25.2\% decrease of phosphoenolpyruvate carboxylase activity was observed during the entire fermentation stage (0-108 h). Results suggested that the combined addition strategy not only enhanced lipid accumulation, but also prevented the lipid peroxidation.
\end{abstract}

Keywords: Schizochytrium sp., Fulvic acid, EDTA, Lipid, Oxidative stress

\section{Introduction}

Microalgae have received growing interest as a potential biofuel feedstock, and are regarded as a promising alternative source for next-generation renewable fuels (Bellou et al. 2014). Microalgal lipids are classified into two types according to their carbon number, with fatty acids containing 14-20 carbons used for biodiesel production, and polyunsaturated fatty acids (PUFA) with more than 20 carbon atoms used as health food supplements. As a heterotrophic oleaginous microalga, Schizochytrium sp. can accumulate over $50 \%$ of lipids with a high fraction of PUFA in its cell dry weight (Ling et al. 2015; Zhao et al. 2018). It consequently received increasing attention as a promising feedstock for the sustainable production of biofuels and food (Markou and Nerantzis 2013). However, cost-effective and biocompatible cultivation

\footnotetext{
*Correspondence: renlujing@njtech.edu.cn

${ }^{1}$ College of Biotechnology and Pharmaceutical Engineering, Nanjing Tech University, No. 30 South Puzhu Road, Nanjing 211816, People's Republic of China

Full list of author information is available at the end of the article
}

strategies for lipid production in Schizochytrium sp. need to be explored further.

Previous studies mainly focus on improving the fermentation process to enhance the lipid content of Schizochytrium sp., and the stress-based strategy is considered as the most successful inducing method (Sahin et al. 2018). However, cellular responses to the stress-based strategy, including low biomass and reactive oxygen species (ROS) formation, are the two major negative effects. The resultant ROS can react with biomacromolecules (such as DNA, lipid and protein) and result in damage, thus leading to loss of protein function and even cell death (Ruenwai et al. 2015). The general countermeasure is to adopt the two-stage culture strategy, apply the optimal growth conditions at the first stage into maximizing the biomass production, and preserve the lipid accumulation at the second stage. For example, the final lipid yield of Nannochloropsis oculata obtained in a two-stage process was 2.82-times higher than that of single-stage cultivation systems (Su et al. 2011). Moreover, many recent studies improved microalgae growth lipid accumulation 
by reduction of oxidative stress by developing high stress tolerant strain and exogenous addition of growth antioxidants. For instance, adaptive evolution under cooperative low temperature and high salinity conditions obtained mutants of Schizochytrium sp. with strong stress tolerance and increased accumulation of lipid rich in PUFA (Sun et al. 2018). In another study, overexpression of superoxide dismutase (SOD) in Schizochytrium sp. also successfully alleviated oxidative stress and increased the PUFA content by 32.9\% (Zhang et al. 2018). Apart from strain development, the addition of antioxidants offers a simple way to alleviate cellular oxidative damage. For instance, the lipid yield and docosahexaenoic acid (DHA) productivity was increased by $14.5 \%$ and $20.0 \%$ in Schizochytrium sp. and Crypthecodinium cohnii by adding ascorbic acid and sesamol, respectively (Liu et al. 2015; Ren et al. 2016). However, the primary effect of antioxidants was only to reduce oxidative damage, rather than induce lipid biosynthesis itself.

It has been reported that plant hormones not only reduce the oxidative damage in cells but also increase the lipid production by controlling internal biochemical pathways (Lu and $\mathrm{Xu} 2015)$, which presents new opportunities for improving microalgal lipid production. As a plant growth regulator, fulvic acid (FA) plays a key role in controlling hormone level and improving the secondary metabolite level (Çimrin et al. 2010). For example, the biomass of Haematococcus pluvialis was increased by $7.13 \%$ and $10.58 \%$ after the addition of 5 and $10 \mathrm{mg} / \mathrm{L}$ FA, respectively (Zhao et al. 2015). Moreover, the highest lipid content can be achieved in Monoraphidium sp. FXY-10 by exogenous addition of $120 \mathrm{mg} / \mathrm{L}$ FA, which is 1.8 times higher compared with the control treatment (Che et al. 2016a). It has been known that ethylene diamine tetraacetic acid (EDTA) is the common component in microalgae growth medium, which can increase the cell permeability through inducing the membrane pore. In recent years, supplementation of EDTA has been also reported to improve the lipid accumulation in microalgae. In Nannochloropsis oculata, the accumulation of biomass and lipids gradually increased with increased concentrations of EDTA (Dou et al. 2013). Moreover, Ren et al. (2014) had also reported that the lipid content in Scenedesmus sp. was markedly increased from 50 to $45 \%$ when the EDTA concentration is increased from 0 to $0.001 \mathrm{~g} / \mathrm{L}$. However, previous studies were focused only on a single phytohormone or EDTA when evaluating their effects on lipid accumulation. Moreover, the precise mechanisms of the improvement of lipid accumulation by exogenous application of phytohormones or EDTA remain elusive.

In this study, the cooperative effect of FA and EDTA on cell growth, lipid productivity, and lipid profile was investigated in Schizochytrium sp. Furthermore, the activities of antioxidant defenses, four key enzymes involved in lipid biosynthesis, as well as the levels of oxidative damage indicators including ROS and MDA, were determined to explore the mechanisms guiding the physiological and molecular changes triggered by FA and EDTA. The aim of this study was to develop a promising strategy to improve lipid productivity, which also might be used in other oil-producing microalgae.

\section{Materials and methods}

\section{Microorganism and culture conditions}

Schizochytrium sp. HX-308 used in this study was isolated from the sea water, which stored in the China Center for Type Cultuer Collection (Number: CCTCC M 209059). The strain was preserved in $20 \%(\mathrm{v} / \mathrm{v})$ glycerinum at $-80{ }^{\circ} \mathrm{C}$. The seed medium and breeding conditions were the same as those used in our previous study (Qu et al. 2011). After three cell passages, the seed culture $(1 \% \mathrm{v} / \mathrm{v})$ was transferred into the $500 \mathrm{~mL}$ shake flask containing $100 \mathrm{~mL}$ medium for shaking culture in the constant tract at $30^{\circ} \mathrm{C}$ at $170 \mathrm{rpm}$. The obtained cell suspension was used as the inoculant for fermentation at inoculum size of $10 \%(\mathrm{v} / \mathrm{v})$. The fermentation medium was the same as that reported in our previous study (Ren et al. 2010). The basic medium were dissolved in artificial sea water, which contained $50 \mathrm{~g} / \mathrm{L}$ glucose and $20 \mathrm{~g} / \mathrm{L}$ monosodium glutamate. One liter of artificial sea water contained (g/L): $0.1 \mathrm{~g} \mathrm{CaCl}_{2}, 10 \mathrm{~g} \mathrm{Na}_{2} \mathrm{SO}_{4}, 4 \mathrm{~g} \mathrm{KH}_{2} \mathrm{PO}_{4}$, $\left.0.8 \mathrm{~g}_{(\mathrm{NH}}\right)_{2} \mathrm{SO}_{4}, 2 \mathrm{~g} \mathrm{MgSO}_{4}, 0.2 \mathrm{~g} \mathrm{KCl}$ and the trance elements: $0.6 \mathrm{~g} \mathrm{CuSO}_{4} \cdot 5 \mathrm{H}_{2} \mathrm{O}, 0.29 \mathrm{~g} \mathrm{FeSO}_{4}, 0.8 \mathrm{~g} \mathrm{ZnSO}_{4}$, $0.01 \mathrm{~g} \mathrm{Na} \mathrm{NoO}_{4} \cdot 2 \mathrm{H}_{2} \mathrm{O}, 0.01 \mathrm{~g} \mathrm{CoCl} \cdot 6 \mathrm{H}_{2} \mathrm{O}, 0.06 \mathrm{~g}$ $\mathrm{NiSO}_{4} \cdot 6 \mathrm{H}_{2} \mathrm{O}$ and $0.86 \mathrm{~g} \mathrm{MnCl}_{2} \cdot 4 \mathrm{H}_{2} \mathrm{O}$.

\section{Determination of cell dry weight and total lipids}

Ten milliliter $(\mathrm{mL})$ culture aliquot was obtained after 5 min of centrifugation at $4500 \times g$, which was used to determine the cell dry weight through gravimetric analysis. Subsequently, the cells were transferred onto the weighing filter paper and dried at $60{ }^{\circ} \mathrm{C}$ to constant weight (about $12 \mathrm{~h}$ ). Meanwhile, the total lipids, extracellular glucose and glutamic acid contents were determined according to the methods used in our previous study (Qu et al. 2011).

\section{Fatty acid analysis}

Fatty acid methyl ester (FAME) was prepared from $0.2 \mathrm{~g}$ dry cells and analyzed using the GC-2010 gas chromatography system (Shimadzu, Japan) equipped with the DB-23 capillary column $(60 \mathrm{~m} \times 0.22 \mathrm{~mm}$; Agilent, USA). Meanwhile, the flame ionization detector (FID) was also employed in this study. Nitrogen was used as the carrier gas. The injection port was maintained at 
$250{ }^{\circ} \mathrm{C}$, with the injection volume of $1 \mu \mathrm{L}$. The column temperature was increased from 100 to $200{ }^{\circ} \mathrm{C}$ at a rate of $2{ }^{\circ} \mathrm{C} / \mathrm{min}$, and to $230{ }^{\circ} \mathrm{C}$ at a rate of $4{ }^{\circ} \mathrm{C} / \mathrm{min}$ and then maintained for $9 \mathrm{~min}$. Temperature of the FID was set at $280^{\circ} \mathrm{C}$. Specifically, FAME was identified through comparing the retention time with the corresponding external true reference standard (Sigma, USA). Besides, nonane-decanoid acid (C19:0) was used as the internal standard to estimate the individual FAME content based on the integral peak area in the chromatogram.

\section{Preparation of cell extracts and enzyme assay}

Microorganisms were obtained through centrifugation, washed with the icy distilled water, and washed with the washing buffer [ $400 \mathrm{mM}$ Tris- $\mathrm{HCl}$ buffer, $\mathrm{pH}$ 7.4 , containing $20 \%(\mathrm{w} / \mathrm{v})$ glycerinum and $1 \mathrm{mM}$ dithiothreitol (DTT)]. Finally, the microorganisms were suspended in the clean washing buffer supplemented with $0.5 \mathrm{mM}$ phenyl-methane-sulfonyl fluoride (PMSF). The material was placed in the ultrasonic knapper for 15 min of crushing, and centrifuged at $4{ }^{\circ} \mathrm{C}$ for $15 \mathrm{~min}$ at $13,800 \times g$, and the supernatant was immediately collected to determine the enzyme activity. Besides, protein was also determined according to the Bradford method using bovine serum albumin (BSA) as the standard. Afterwards, the total protein content $(\mu \mathrm{g} / \mathrm{mL})$ in the crude enzyme was calculated according to the empirical calibration equation (protein content $\left.=0.1833 \mathrm{OD}_{595 \mathrm{~nm}}-0.0033\right)$.

Malic enzyme (ME) and glucose-6-phosphate dehydrogenase $(\mathrm{G} 6 \mathrm{PDH})$ activities was determined spectrophotometrically by monitoring the rate of NADPH formation at $28{ }^{\circ} \mathrm{C}$ and $25{ }^{\circ} \mathrm{C}$, respectively. SOD and catalase (CAT) activities were determined using an assay kit (Nanjing Jiancheng Bioengineering Institute, China) according to the manufacturer's instructions. The enzyme activity unit (U) was defined as the quantitative formation of $\mathrm{NADPH} / \mathrm{min}$, which equaled to $0.001 / \mathrm{min}$ increase at $\mathrm{OD}_{340 \mathrm{~nm}}$. The specific activity (U/ $\mathrm{mg}$ protein) was defined as the activity unit/mg protein.

\section{Determination of intracellular reactive oxygen species and lipid peroxidation}

The types of ROS were determined in accordance with the same methods reported in our previous study (Ren et al. 2016). According to the method proposed by Heath and Packer (1968), the malondialdehyde (MDA) equivalent was measured to determine the lipid peroxidation level. Additionally, the treated and untreated cells were homogenized in $5 \%(\mathrm{w} / \mathrm{v})$ TCA and centrifuged at $10,000 \times g$ for $10 \mathrm{~min}$ at $4{ }^{\circ} \mathrm{C}$. The reaction mixture containing the thiobarbituric acid-TCA solution and cell extracts were boiled for $20 \mathrm{~min}$; later, the absorbance of the reaction mixture was recorded at 532 and $600 \mathrm{~nm}$. The absorbance value recorded at $532 \mathrm{~nm}$ was subtracted by the non-specific absorbance value recorded at $600 \mathrm{~nm}$, and the MDA equivalent content was measured using the $155 \mathrm{mM} / \mathrm{cm}$ extinction coefficient.

\section{Results}

Effect of FA on biomass and lipid production

Addition of FA at various concentrations $(0,10,15,20$, 25 and $30 \mathrm{mg} / \mathrm{L}$ ) into the medium would result in markedly changes in the cell growth and lipid accumulation of Schizochytrium sp. As shown in Table 1, the increase in FA concentration had given rise to the remarkable increases in cell dry weight (CDW) and lipid production. The optimal FA concentration was $20 \mathrm{mg} / \mathrm{L}$, and the maximum CDW at that concentration was $47.7 \mathrm{~g} / \mathrm{L}$, while the lipid production was $16.4 \mathrm{~g} / \mathrm{L}$, which were $23.3 \%$ and $34.4 \%$ higher than those in the non-supplementation group, respectively. Such results were consistent with those reported in previous studies, namely, FA had the potential to enhance the lipid production in Monoraphidium sp. FXY-10 (Che et al. 2016b). Moreover, FA can increase the tolerance of microalgae to biotic and abiotic stresses, which might contribute to better cell growth and counteract the drop of microalgal productivity during prolonged fermentation. The maximum lipid productivity of $0.41 \mathrm{~g} / \mathrm{L} / \mathrm{h}$ was obtained in the group supplemented with $20 \mathrm{mg} / \mathrm{L}$ FA. However, a further increase in FA concentration to 25 and $30 \mathrm{mg} / \mathrm{L}$ resulted in a drop

Table 1 Cell growth and lipid production of Schizochytrium sp. under different concentrations of fulvic acid

\begin{tabular}{lllllll}
\hline & \multicolumn{7}{l}{ FA concentration $(\mathbf{m g} / \mathrm{L})$} & & & \\
\cline { 2 - 7 } & $\mathbf{0}$ & $\mathbf{1 0}$ & $\mathbf{1 5}$ & $\mathbf{2 0}$ & $\mathbf{2 5}$ & $\mathbf{3 0}$ \\
\hline $\mathrm{CDW}(\mathrm{g} / \mathrm{L})$ & $38.7 \pm 0.7$ & $39.9 \pm 0.6$ & $42.4 \pm 0.8$ & $47.7 \pm 0.5$ & $40.5 \pm 0.7$ & $34.2 \pm 0.5$ \\
Lipid yield $(\mathrm{g} / \mathrm{L})$ & $12.2 \pm 0.3$ & $12.5 \pm 0.2$ & $12.9 \pm 0.1$ & $16.4 \pm 0.3$ & $13.6 \pm 0.4$ & $9.7 \pm 0.3$ \\
Fermentation time $(\mathrm{h})$ & 45 & 42 & 40 & 40 & 45 & 50 \\
Lipid productivity $(\mathrm{g} / \mathrm{L} / \mathrm{h})$ & 0.27 & 0.30 & 0.32 & 0.41 & 0.30 & 0.19 \\
\hline
\end{tabular}

Data represent the mean values and standard deviations of three replicates for each measurement 
of CDW and lipid productivity, suggested that high FA concentrations (over $20 \mathrm{mg} / \mathrm{L}$ ) might actually hinder the growth of Schizochytrium sp. The biochemical basis remained unclear, but previous studies had verified that, high concentration of plant growth regulator 2,4-dichlorophenoxyacetic acid would also suppress cell proliferation (Saygideger and Okkay 2008).

\section{Cumulative effects of FA and EDTA on biomass and lipid production}

In order to enhance the availability of FA or nutrients to Schizochytrium sp., EDTA was added into the medium in concentrations ranging from 0.1 to $2.0 \mathrm{~g} / \mathrm{L}$ in addition to the supplementation with $20 \mathrm{mg} / \mathrm{L}$ FA (Table 2). At EDTA concentrations from 0.1 to $1.0 \mathrm{~g} / \mathrm{L}$, the lipid yield increased along with a higher lipid productivity of $0.52 \mathrm{~g} / \mathrm{L} / \mathrm{h}$, representing $26.8 \%$ and $92.6 \%$ increases over the supplementation of $20 \mathrm{mg} / \mathrm{L}$ FA without EDTA supplemented and no supplementation whatsoever, respectively. However, further increase in EDTA concentration to $2.0 \mathrm{~g} / \mathrm{L}$ could also dramatically suppress microalgae growth, and the final lipid production rate was only $0.35 \mathrm{~g} / \mathrm{L} / \mathrm{h}$ upon the end of the experiment. High EDTA concentration was well-known to strongly chelate metal ions (Kern et al. 1992), suggesting that high EDTA level would distinctly reduce the availability of metal ions essential for the growth of microalgae, leading to low total lipid production. Similarly, Ren et al. (2014) had reported that, further increase in EDTA concentration to $1.0 \mathrm{~g} / \mathrm{L}$ would apparently restrain the growth of microalgae Scenedesmus sp. Therefore, the addition of $20 \mathrm{mg} / \mathrm{L}$ FA and $1.0 \mathrm{~g} / \mathrm{L}$ EDTA was the most promising strategy for lipid accumulation in Schizochytrium sp.

\section{The batch-fermentation behavior of Schizochytrium sp. with the combined addition strategy}

To further investigate the practicability of the combined addition strategy $(20 \mathrm{mg} / \mathrm{L} \mathrm{FA}$ and $1.0 \mathrm{~g} / \mathrm{L}$ EDTA) in the production of lipids, we used it in conjunction with conventional culture in a 5-L bioreactor. As shown in Fig. 1a, a significant difference in glucose consumption was identified between the non-supplemented cultures and those supplemented with $20 \mathrm{mg} / \mathrm{L}$ FA and $1.0 \mathrm{~g} / \mathrm{L}$ EDTA. Compared to the control group, Schizochytrium sp. exhibited faster substrate consumption with the combined addition strategy, which might be attributed to higher cell permeability induced by EDTA. Moreover, FA can also improve cell growth by controlling internal biochemical pathways. The cooperative effect of two chemicals resulted the highest CDW of $130.7 \mathrm{~g} / \mathrm{L}$ at the end of the fermentation, which was $36.4 \%$ higher than that of the control group (Fig. 1b).

The total lipid yield increased sharply in both groups after nitrogen exhaustion $(36 \mathrm{~h})$. A maximum yield corresponding to $80.4 \mathrm{~g} / \mathrm{L}$ of total lipids was obtained with the combined addition strategy, which was $52.9 \%$ higher than that of the control group (Table 3). In has been reported that FA can increase lipid production by enhancing the degradation of carbohydrates to biosynthesis lipids (Che et al. 2016b), which is consistent with the higher glucose consumption observed with the combined addition strategy. Moreover, as shown in Fig. 1c, the lipid productivity increased with culture time in both groups, reached its maximum at $84 \mathrm{~h}$ and then declined at later fermentation stages. Compared with the control group, the combined addition group exhibited an overall higher lipid productivity during the entire fermentation period, especially during the later fermentation stage $(>72 \mathrm{~h})$. At the end of fermentation, the lipid productivity reached $1.16 \mathrm{~g} / \mathrm{L} / \mathrm{h}$ with the combined addition strategy, which was about threefold higher than that of the control group. These results were in contrast with several reports on the application of other plant growth regulators, such as jasmonic acid and indole-3-acetic acid, which induced lipid accumulation only during the early growth phase (Jusoh et al. 2015).

Interestingly, addition of FA and EDTA would not only markedly affect cell growth and lipid production, but would also significantly influence the fatty acid composition. The saturated fatty acid (SFA) in Schizochytrium sp.

Table 2 Cell growth and lipid production of Schizochytrium sp. under different concentrations of EDTA on the premise of adding $20 \mathrm{mg} / \mathrm{L}$ fulvic acid

\begin{tabular}{llllll}
\hline & \multicolumn{2}{l}{$\mathbf{2 0} \mathbf{~ m g / L ~ F A + E D T A ~ c o n c e n t r a t i o n ~ ( g / L ) ~}$} & & \\
\cline { 2 - 6 } & $\mathbf{0 . 1}$ & $\mathbf{0 . 2}$ & $\mathbf{0 . 5}$ & $\mathbf{1 . 0}$ & $\mathbf{2 . 0}$ \\
\hline CDW (g/L) & $47.2 \pm 0.3$ & $46.8 \pm 0.6$ & $50.1 \pm 0.4$ & $51.4 \pm 0.3$ & $46.5 \pm 0.5$ \\
Lipid yield (g/L) & $16.2 \pm 0.7$ & $17.6 \pm 0.3$ & $18.8 \pm 0.6$ & $20.6 \pm 0.5$ & $15.8 \pm 0.4$ \\
Fermentation time (h) & 40 & 40 & 40 & 40 & 45 \\
Lipid productivity (g/L/h) & 0.41 & 0.44 & 0.47 & 0.52 \\
\hline
\end{tabular}

Data represent the mean values and standard deviations of three replicates for each measurement 

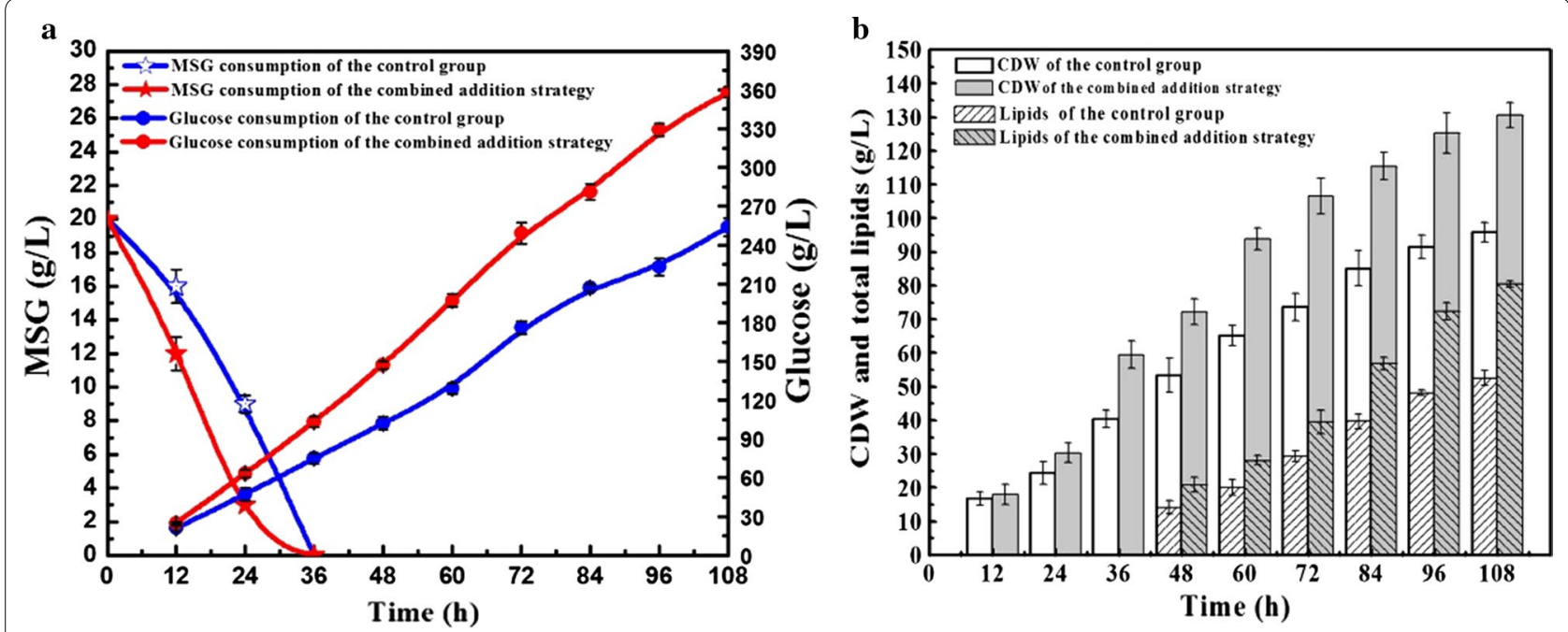

c

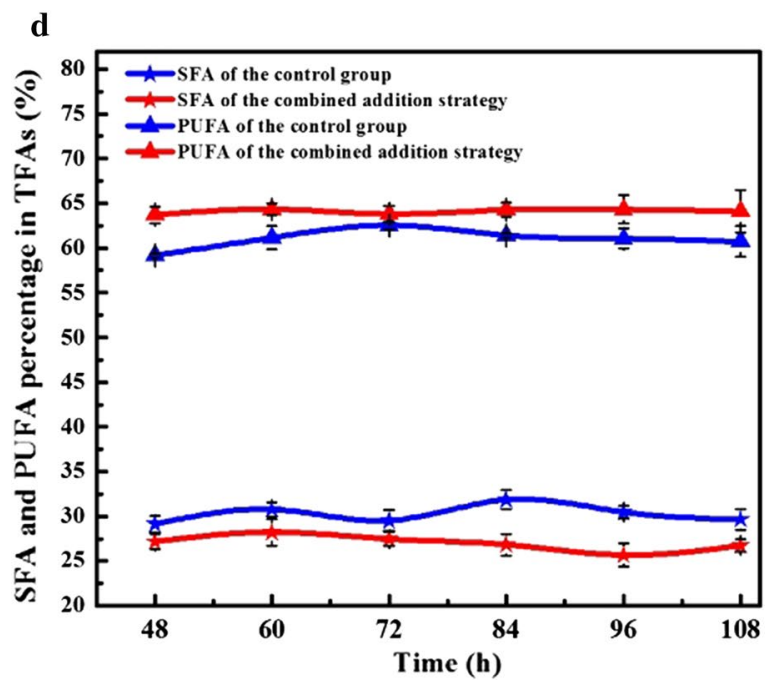

Fig. 1 Comparison between the control group and the combined addition strategy in a 5-L bioreactor: a substrate consumption, b CDW and lipid content, $\mathbf{c}$ lipid productivity, $\mathbf{d}$ SFA and PUFA percentage in TFAs. Values and error bars represent the means and standard deviations from triplicate experiments

Table 3 Fermentation results of the combined strategy in a $5 \mathbf{L}$ bioreactor and \% changes compared to the same results from the control group

\begin{tabular}{lllc}
\hline Parameters & The control group & The combined addition strategy & Increase (\%) \\
\hline CDW (g/L) & $95.8 \pm 2.8$ & $130.7 \pm 3.7$ & 36.4 \\
Lipid yield (g/L) & $52.6 \pm 2.3$ & $80.4 \pm 0.9$ & 52.9 \\
Lipid in CDW (W/W) & 0.55 & 0.62 & 12.7 \\
Lipid productivity (g/L/h) & 0.49 & 0.74 & 51.0 \\
SFA percentage (\% TFAs) & $29.7 \pm 1.2$ & $26.8 \pm 0.7$ & -9.8 \\
PUFA percentage (\% TFAs) & $60.7 \pm 1.7$ & $64.1 \pm 2.4$ & 5.6
\end{tabular}

The control group: no-addition of FA and EDTA; the combined addition strategy: addition of $20 \mathrm{mg} / \mathrm{L} \mathrm{FA}$ and $1.0 \mathrm{~g} / \mathrm{L}$ EDTA. Data represent the mean values and standard deviations of three replicates for each measurement 
was mainly composed of C14:0 and C16:0, while the polyunsaturated fatty acid (PUFA) was mainly comprised of docosenoic acid (DPA) and docosahexaenoic acid (DHA). As shown in Fig. 1d, the SFA and PUFA biosynthesis values were inversely related in both cultures. Notably, the total fatty acid (TFA) and SFA percentages in the cultures supplemented with FA and EDTA were always lower than those in the control. By contrast, the PUFA percentage in TFAs increased in cultures supplemented with $20 \mathrm{mg} / \mathrm{L}$ FA and $1.0 \mathrm{~g} / \mathrm{L}$ EDTA, resulting in 64.1\% PUFA in TFAs. Moreover, a decline of the PUFA percentage was observed in the control group after $60 \mathrm{~h}$. PUFA are wellrecognized antioxidants, and a large number of studies had been carried out focusing on their production and use (Richard et al. 2008). After 60 h, Schizochytrium sp. might begin to consume the PUFA to protect itself from oxidative injury, and such secondary consumption might account for the reduction in PUFA percentage. Accordingly, the combined addition strategy safeguarded an overall better PUFA percentage, which implied that FA and EDTA can alleviate the oxidative damage typical for later fermentation stages.

\section{Influence of the combined addition strategy on the anti-oxidative defense systems for Schizochytrium} sp.

In order to investigate the effects of FA and EDTA on the oxidative defense systems of Schizochytrium sp., the concentrations of ROS and MDA, as well as the activities of antioxidant enzymes were determined. Cellular ROS mainly include the superoxide anion $\left(\mathrm{O}_{2}^{-}\right)$, hydrogen peroxide $\left(\mathrm{H}_{2} \mathrm{O}_{2}\right)$, hydroxyl radical (.OH), lipid hydroperoxides (LOOH), and peroxyl radicals (LOO.) (Zhang et al. 2017). During the initial stage $(<60 \mathrm{~h})$, Schizochytrium sp. cells were in the exponential growth phase and were able to produce ample antioxidants to resist oxidative stress. Consequently, both cultures maintained low ROS levels during this stage (Fig. 2a). Moreover, lipid peroxidation is another commonly used stress maker, which can be measured through detecting the MDA content in cell (Heath et al. 1968). Similar to ROS levels, the MDA levels also sharply increased in both cultures after $60 \mathrm{~h}$ (Fig. 2b). Conversely, the addition of FA and EDTA reduced the ROS and MDA levels, especially after $60 \mathrm{~h}$. The ROS levels in the supplemented group were reduced by $22.5 \%$ at $12 \mathrm{~h}$ and remained significantly lower at $108 \mathrm{~h}$ compared to the non-supplemented control group. By the end of fermentation, a minimum MDA level of $70.3 \mathrm{mmol} / \mathrm{g} \mathrm{Fw}$ was measured in the culture using the combined addition strategy, which was $26.9 \%$ lower than that of the control group (Fig. 2b). In contrast, an overall strong SOD and CAT activity was observed in the culture utilizing the combined addition strategy (Fig. 2c, d).
For example, by the end of fermentation, SOD and CAT activity reached up to 423.5 and $175.2 \mathrm{U} / \mathrm{mg}$ protein, which was $66.7 \%$ and $81.9 \%$ higher than that of the control group, respectively (Fig. 2c, d).

\section{Influence of the combined addition strategy on the activities of key enzymes of Schizochytrium sp.}

In order to further investigate the regulatory mechanisms mediating the observed effects of FA and EDTA on lipid production, the activities of key enzymes related to lipid accumulation (G6PDH, ME, ACCase, and PEPC) were detected every $24 \mathrm{~h}$ during the entire fermentation process. As shown in Fig. 3a, the activity of G6PDH increased in both cultures with increasing culture time and reached its maximum at $36 \mathrm{~h}$, with no obvious differences. However, the combined addition strategy led to an overall higher $\mathrm{ME}$ activity than that of the control group. The ME activity reached $291.3 \mathrm{U} / \mathrm{mg}$ protein at $60 \mathrm{~h}$, representing an increase of $36.6 \%$ over the control (Fig. 3b). In addition, the time point of the maximum value of ME activity $(60 \mathrm{~h})$ lagged far behind the time point of highest G6PDH activity (36 h). This illustrates that G6PDH is responsible for the NADPH supply during the early fermentation stages, while ME mainly functions to resolve the insufficiency of NADPH supply during the later stages.

Moreover, the addition of FA and EDTA resulted in a reduction of ACCase activity to $88.5 \mathrm{U} / \mathrm{mg}$ protein at $108 \mathrm{~h}$, which was $18.1 \%$ lower than the corresponding value of the control group (Fig. 3c). One of the ROS species, the hydroxyl radical $(\cdot \mathrm{OH})$, can react with bicarbonate to form an intermediate bicarbonate radical, which is a crucial step needed at the active site of the ACCase enzyme (Menon et al. 2013). In this study, the lower ROS levels might have decreased the ACCase activity in the culture with the combined addition strategy. Similarly, compared to the control group, a 19.4-25.2\% decrease of PEPC activity was observed with the combined addition strategy during the entire fermentation process (Fig. 3d).

\section{Discussion}

As a kind of aerobiotic microalgae, ROS production is an inevitable aspect for Schizochytrium sp. Moreover, lipids, especially PUFA, can be oxidized themselves (Niki 2009), and the lipid peroxidation can also lead to ROS accumulation at a high level. However, microalgae has evolved an adaptive approach to eliminate and neutralize the ROS; for instance, the application of a variety of antioxidant enzymes. Therefore, it is important to control the balance between oxidative injury and antioxidant ability in Schizochytrium sp. for lipid accumulation. In fact, nitrogen starvation can induce oxidative damage to cells, but this negative effect on exponential phase is greatly lower 

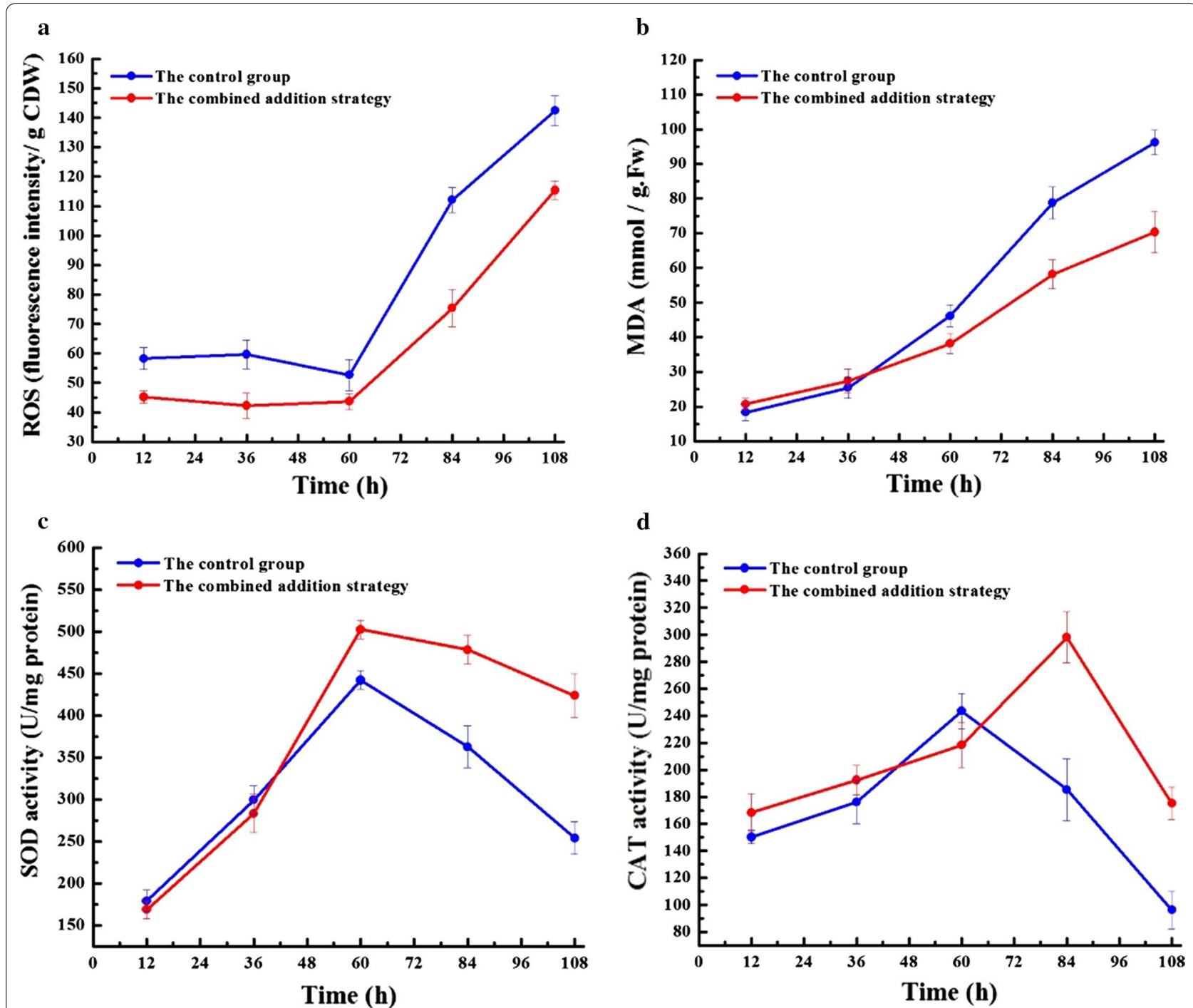

Fig. 2 Comparison of anti-oxidative defense system between the control group and the combined addition strategy in a 5-L bioreactor: a ROS levels, b MDA levels, c SOD activity, d CAT activity. Values and error bars represent the means and standard deviations from triplicate experiments

than stationary phase, because cells at exponential phase can product lots of stress factors to alleviate the oxidative damage (Liu et al. 2012). As shown in Fig. 1a, nitrogen (MSG) was depleted at $36 \mathrm{~h}$, but the ROS levels was decreased at $36-60 \mathrm{~h}$ (Fig. 2a), which might attribute to the production of antioxidants by Schizochytrium sp. After $60 \mathrm{~h}$, with the rapid accumulation of lipid, lipid peroxidation could result in high level of ROS till the end of fermentation (Ruenwai et al. 2015). During this stage, cells begin the experience oxidative stress, since the amount of ROS has exceeded the antidotic capacity of the antioxidant defense system. ROS accumulation can induce oxidative injury to the cellular components, and exert adverse influence on cell growth, which may explain for the low CDW and the reduced PUFA percentage in the non-supplemented group (Fig. 1b, d).

Nonetheless, during the whole fermentation process, relatively low ROS level while high SOD and CAT activities are maintained in the supplemented group (Fig. 2c, d), suggesting that the combined addition strategy can mitigate oxidative injury through enhancing the activity of antioxidant enzymes. Similarly, some evidence indicates that plant hormone can regulate the oxidative stress response in microalgae (Han et al. 2018). For instance, the addition of abscisic acid can greatly reduce the oxidative stress in Chlamydomonas reinhardtii through enhancing the CAT and APX activities. Consistently, in Chlorella vulgaris, the greatest enhancements 

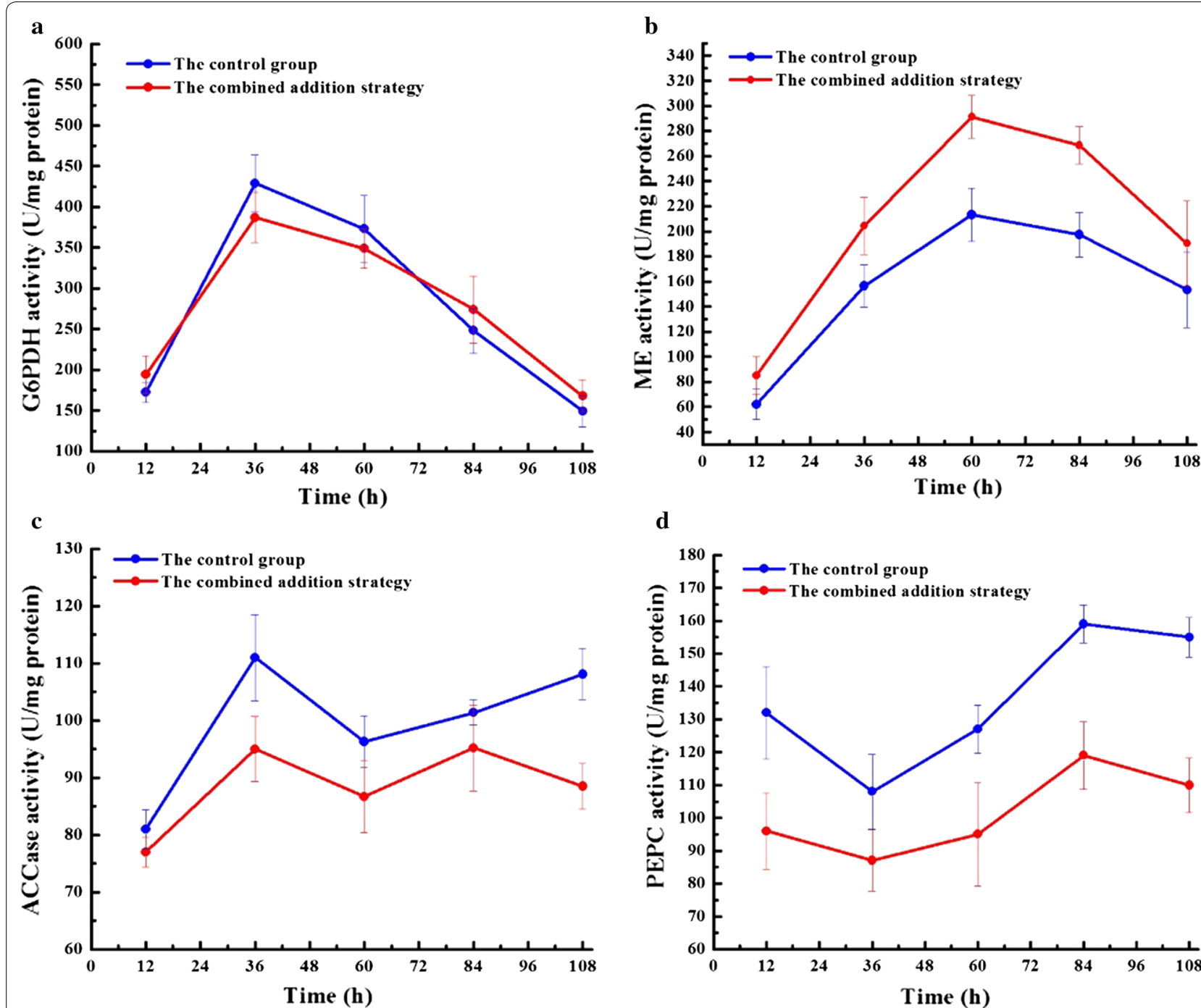

d

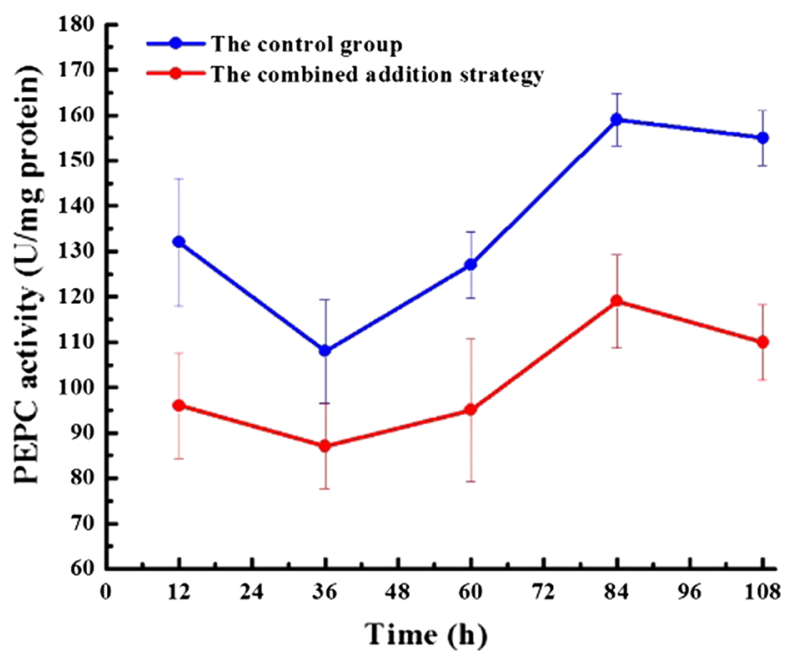

Fig. 3 Comparison of key enzymes involved lipid biosynthesis between the control group and the combined addition strategy in a 5-L bioreactor: a G6PDH activity, b ME activity, c ACCase activity, d PEPC activity. Values and error bars represent the means and standard deviations from triplicate experiments

in antioxidant activities (55\% for SOD, $89 \%$ for CAT, and $75 \%$ for APX) can be attributed to the addition of indol-3-acetic acid (Piotrowska-Niczyporuk and Bajguz 2014). Actually, plant hormone is generally related to the ROS-induced signal transduction. For example, the mitogen-activated protein kinase (MAPK)-activated $\mathrm{H}_{2} \mathrm{O}_{2}$ treatment can down-regulate the expression of auxin response gene (Kovtun et al. 2000). Recently, Beck et al. (2007) had proposed that abscisic acid could participate in the ROS signaling pathway, and regulated ROS production and clearance, which might be a potential oxidative stress regulatory mechanism. Interestingly, the MDA level is markedly reduced in the combined addition strategy, demonstrating that FA and EDTA can not only enhance lipid accumulation, but can also prevent lipid loss resulted from peroxidation.

As is well known, the reducing energy provided in the form of NADPH plays a key role in lipid biosynthesis (Tan and Lee 2016), while the transhydrogenase circulation and pentose phosphate pathway (PPP) are the two major NADPH regeneration pathways in Schizochytrium sp. Of them, G6PDH and ME are the respective key enzymes. In this study, the combined addition strategy has resulted in the overall higher ME activity than that in the control group, while G6PDH activity is not markedly changed (Fig. 3a, b). These results indicate that, compared with ME, G6PDH only plays a mild role in the lipid accumulated NADPH supply. Such phenomenon can 
also be observed in the microalgae Aurantiochytrium sp. (Song et al. 2013). Interestingly, the latest research indicates that, the PPP pathways can bind with the polyketide synthase (PKS) to provide NADPH for the biosynthesis of PUFA, whereas the transhydrogenase system can couple with the FAS pathway to provide NADPH for SFA biosynthesis (Beopoulos et al. 2011; Liang and Jiang 2015). Over-expression of G6PDH gene in Aurantiochytrium sp can successfully improve the PUFA percentage by $10.6 \%$ (Cui et al. 2016). However, overexpression of G6PDH gene in Phaeodactylum tricornutum would lead to reduction in PUFA proportion by about $22.7 \%$, although the lipid content is increased by 2.7 times (Xue et al. 2017). Such difference may be attributed to the fact that Aurantiochytrium sp has synthesized PUFA through the PKS pathway, while Phaeodactylum tricornutum has adopted the desaturase/elongase pathway. In Schizochytrium sp., the FAS enzyme complex is of crucial importance to the synthesis of SFA, while the PKS pathway is responsible for PUFA synthesis (Ren et al. 2010). In this study, the SFA percentage is not increased, but the combined addition strategy has increased the ME activity, indicating that FA and EDTA can more effectively produce the total lipids, rather than change the fatty acid profile. In recent years, many studies used chemical regulators to improve biomass and lipid accumulation of thraustochytrids. In the previous study, the CDW and lipid yield of Schizochytrium sp. can be increased to $106.7 \mathrm{~g} / \mathrm{L}$ and $65.5 \mathrm{~g} / \mathrm{L}$ after addition of $9 \mathrm{~g} / \mathrm{L}$ of ascorbic acid, which was $16.2 \%$ and $30.4 \%$ higher than that of the non-supplemented group, respectively (Ren et al. 2016). Moreover, in Aurantiochytrium sp. YLH70, addition of $4 \mathrm{mg} / \mathrm{mL}$ of gibberellin resulted in the maximum biomass of $21.4 \mathrm{~g} / \mathrm{L}$ and lipid yield of $11.6 \mathrm{~g} / \mathrm{L}$, representing a $14.4 \%$ and $43.6 \%$ higher than that of the non-supplemented group, respectively (Yu et al. 2016).

Acetyl-CoA has been well recognized to be the major precursor for fatty acid biosynthesis, while the acetyl-CoA carboxylase (ACCase) and PEPC are the key enzymes related to the carbon precursor supply. ACCase can catalyze the first step of fatty acid biosynthesis, namely, the transformation of acetyl-CoA to malonyl-CoA, which is also the rate-limiting step of fatty acid biosynthesis (Davis et al. 2000). In this study, the combined addition strategy has restricted the ACCase activity, which is theoretically contradictory to the high lipid productivity. However, it is found in another study that, addition of FA can increase the ACCase activity by three times in Monoraphidium sp. FXY-10 (Che et al. 2016b) suggests that the action of FA depends on the microalgae species. Actually, almost no attempt to improve ACCase activity to improve lipid productivity is successful. For instance, in Nannochloropsis oceanica and Neochloris oleoabundans, the expression of ACCase gene is greatly reduced under nitrogen deprivation, but the total lipid content is outstandingly increased ( $\mathrm{Li}$ and $\mathrm{Xu}$ 2014; Rismani-Yazdi et al. 2012). PEPC can irreversibly catalyze the carboxylation of phosphoenolpyruvic acid into oxaloacetic acid, and introduce the metabolic flux into the Krebs circulation, which can provide the substrates and energy for cell growth, thus reducing the carbon flux towards lipid biosynthesis (Tian et al. 2014). Consequently, suppressing PEPC to promote the carbon flux towards fatty acid biosynthesis can promote lipid production, which is consistent with the reduced PEPC activity observed in this study (Fig. 3d). Such result is highly consistent with that in another study (Che et al. 2016b). As a result, PEPC knockdown in Chlamydomonas reinhardtii and Thalassiosira pseudonana can increase the TAG level by $74.4 \%$ and four times, respectively (Trentacoste et al. 2013; Kao and Ng 2017).

In conclusion, the strategy of adding $20 \mathrm{mg} / \mathrm{L} \mathrm{FA}$ and $1.0 \mathrm{~g} / \mathrm{L}$ EDTA is developed in this study to induce lipid biosynthesis and reduce oxidative injury. Meanwhile, the effects of the combined strategy on cell growth, lipid production, and lipid profile were investigated. Results indicated that cell growth and lipid yield were sharply increased by $36.4 \%$ and $52.9 \%$, respectively. These major performance changes were accompanying with the reduced ROS level and enhanced antioxidant enzyme activities, proving that the combined addition strategy can effectively alleviate cell oxidative injury. Moreover, ME activity was considerably increased, whereas PEPC activity was significantly decreased. These findings have illustrated the potential molecular changes for the excessive lipid production related to the combined addition strategy.

\section{Abbreviations}

ACCase: acetyl-CoA carboxylase; CAT: catalase; CDW: cell dry weight; EDTA: ethylenediaminetetraacetic acid; FA: fulvic acid; PEPC: phosphoenolpyruvate carboxylase; PUFA: polyunsaturated fatty acids; ROS: reactive oxygen species; SFA: saturated fatty acids; SOD: superoxide dismutase.

\section{Authors' contributions}

LJR and HH designed the experiments. XMS and XJJ performed the research. XMS and LJR analyzed the data. HH supervised the research. XMS wrote the paper. XJJ polished the paper. All authors read and approved the final manuscript.

\footnotetext{
Author details

${ }^{1}$ College of Biotechnology and Pharmaceutical Engineering, Nanjing Tech University, No. 30 South Puzhu Road, Nanjing 211816, People's Republic of China. ${ }^{2}$ School of Pharmaceutical Sciences, Nanjing Tech University, No. 30 South Puzhu Road, Nanjing 211816, People's Republic of China. ${ }^{3}$ State Key Laboratory of Materials-Oriented Chemical Engineering, Nanjing Tech University, No. 5 Xinmofan Road, Nanjing 210009, People's Republic of China. 4 Jiangsu National Synergetic Innovation Center for Advanced Materials (SICAM), Nanjing, People's Republic of China.
} 


\section{Acknowledgements}

We are grateful to Dr.rer.nat. Ivan Hajnal for critical reading and editing of our manuscript.

\section{Competing interests}

The authors declare that they have no competing interests.

\section{Availability of data and materials}

All data generated or analyzed during this study are included in the figures and tables. Any material used in this study is available for research purposes upon request.

\section{Consent for publication}

Not applicable.

\section{Ethics approval and consent to participate}

Not applicable.

\section{Funding}

This work was financially supported by the Outstanding Youth Foundation of Jiangsu Natural Science Foundation (BK20160092), the Program for Innovative Research Teams in Universities of Jiangsu Province (2015), and the Jiangsu Synergetic Innovation Center for Advanced Bio-Manufacture (XTE1829).

\section{Publisher's Note}

Springer Nature remains neutral with regard to jurisdictional claims in published maps and institutional affiliations.

Received: 10 July 2018 Accepted: 18 September 2018 Published online: 21 September 2018

\section{References}

Beck EH, Fettig S, Knake C, Hartig K, Bhattarai T (2007) Specific and unspecific responses of plants to cold and drought stress. J Biosci 37:501-510

Bellou S, Baeshen MN, Elazzazy AM, Aggeli D, Sayegh F, Aggelis G (2014) Microalgal lipids biochemistry and biotechnological perspectives. Biotechnol Adv 32:1476-1493

Beopoulos A, Nicaud JM, Gaillardin C (2011) An overview of lipid metabolism in yeasts and its impact on biotechnological processes. Appl Microbiol Biotechnol 90:1193-1206

Che R, Ding K, Huang L, Zhao P, Xu JW, Li T, Ma H, Yu X (2016a) Enhancing biomass and oil accumulation of Monoraphidium sp. FXY-10 by combined fulvic acid and two-step cultivation. J Taiwan Inst Chem E 67:161-165

Che R, Li H, Xu JW, Peng Z, Tao L, Ma H, Yu X (2016b) Effect of fulvic acid induction on the physiology, metabolism, and lipid biosynthesis-related gene transcription of Monoraphidium sp. FXY-10. Bioresour Technol 227:324-334

Cimrin KM, Türkmen Ö, Turan M, Tuncer B (2010) Phosphorus and humic acid application alleviate salinity stress of pepper seedling. Afr J Biotechnol 9:5845-5851

Cui GZ, Ma Z, Liu YJ, Feng Y, Sun Z, Cheng Y, Song X, Cui Q (2016) Overexpression of glucose-6-phosphate dehydrogenase enhanced the polyunsaturated fatty acid composition of Aurantiochytrium sp. SD116. Algal Res 19:138-145

Davis MS, Solbiati J, Cronan JE (2000) Overproduction of acetyl-CoA carboxylase activity increases the rate of fatty acid biosynthesis in Escherichia coli. J Biol Chem 275:28593-28598

Dou X, Lu XH, Lu MZ, Yu LS, Xue R, Ji JB (2013) The effects of trace elements on the lipid productivity and fatty acid composition of Nannochloropis oculata. J Renew Energy 2013:1-6. https://doi.org/10.1155/2013/671545

Han X, Zeng H, Bartocci P, Fantozzi F, Yan Y (2018) Phytohormones and effects on growth and metabolites of microalgae: a review. Fermentation 4(2):25

Heath RL, Packer L (1968) Photoperoxidation in isolated chloroplasts. I. Kinetics and stoichiometry of fatty acid peroxidation. Arch Biochem Biophys 125:189-198

Jusoh M, Loh SH, Chuah TS, Aziz A, Cha TS (2015) Elucidating the role of jasmonic acid in oil accumulation, fatty acid composition and gene expression in Chlorella vulgaris (Trebouxiophyceae) during early stationary growth phase. Algal Res 9:14-20

Kao PH, Ng IS (2017) CRISPRi mediated phosphoenolpyruvate carboxylase regulation to enhance the production of lipid in Chlamydomonas reinhardtii. Bioresour Technol 245:1527-1537

Kern M, Koch HG, Klemme JH (1992) EDTA activation of $\mathrm{H}_{2}$ photoproduction by Rhodospirillum rubrum. Appl Microbiol Biotechnol 37:496-500

Kovtun Y, Chiu W, Tena G, Sheen J (2000) Functional analysis of oxidative stressactivated mitogen-activated protein kinase cascade in plants. Proc Natl Acad Sci USA 97:2940-2945

Li J, Xu J (2014) Choreography of transcriptomes and lipidomes of Nannochloropsis reveals the mechanisms of oil synthesis in microalgae. Plant Cell 26:1645-1665

Liang YJ, Jiang JG (2015) Characterization of malic enzyme and the regulation of its activity and metabolic engineering on lipid production. RSC Adv 5:45558-45570

Ling X, Guo J, Liu X, Zhang X, Wang N, Lu Y, Ng IS (2015) Impact of carbon and nitrogen feeding strategy on high production of biomass and docosahexaenoic acid (DHA) by Schizochytrium sp. LU310. Bioresour Technol 184:139-147

Liu WH, Huang ZW, Li P, Xia JF, Chen B (2012) Formation of triacylglycerol in Nitzschia closterium $\mathrm{f}$. minutissima under nitrogen limitation and possible physiological and biochemical mechanisms. J Exp Mar Biol Ecol 418:24-29

Liu B, Jin L, Sun P, Ma X, Yue J, Feng C (2015) Sesamol enhances cell growth and the biosynthesis and accumulation of docosahexaenoic acid in the microalga Crypthecodinium cohnii. J Agric Food Chem 63:5640-5645

Lu Y, Xu J (2015) Phytohormones in microalgae: a new opportunity for microalgal biotechnology? Trends Plant Sci 20:273-282

Markou G, Nerantzis E (2013) Microalgae for high-value compounds and biofuels production: a review with focus on cultivation under stress conditions. Biotechnol Adv 31:1532-1542

Menon KR, Balan R, Suraishkumar GK (2013) Stress induced lipid production in Chlorella vulgaris: relationship with specific intracellular reactive species levels. Biotechnol Bioeng 110:1627-1636

Niki E (2009) Lipid peroxidation: physiological levels and dual biological effects. Free Radic Biol Med 47:469-484

Piotrowska-Niczyporuk A, Bajguz A (2014) The effect of natural and synthetic auxins on the growth, metabolite content and antioxidant response of green alga Chlorella vulgaris (Trebouxiophyceae). Plant Growth Regul 73:57-66

Qu L, Ji XJ, Ren LJ, Nie ZK, Feng Y, Wu WJ, Ouyang PK, Huang H (2011) Enhancement of docosahexaenoic acid production by Schizochytrium sp. using a two-stage oxygen supply control strategy based on oxygen transfer coefficient. Lett Appl Microbiol 52:22-27

Ren LJ, Ji XJ, Huang H, Qu L, Feng Y, Tong QQ, Ouyang PK (2010) Development of a stepwise aeration control strategy for efficient docosahexaenoic acid production by Schizochytrium sp. Appl Microbiol Biotechnol 87:1649-1656

Ren HY, Liu BF, Kong F, Zhao L, Xie GJ, Ren NQ (2014) Enhanced lipid accumulation of green microalga Scenedesmus sp. by metal ions and EDTA addition. Bioresour Technol 169:763-767

Ren LJ, Sun XM, Ji XJ, Chen SL, Guo DS, Huang H (2016) Enhancement of docosahexaenoic acid synthesis by manipulation of antioxidant capacity and prevention of oxidative damage in Schizochytrium sp. Bioresour Technol 223:141-148

Richard D, Kefi K, Barbe U, Bausero P, Visioli F (2008) Polyunsaturated fatty acids as antioxidants. Pharmacol Res 57:451-455

Rismani-Yazdi H, Haznedaroglu BZ, Hsin C, Peccia J (2012) Transcriptomic analysis of the oleaginous microalga Neochloris oleoabundans reveals metabolic insights into triacylglyceride accumulation. Biotechnol Biofuels $5: 1-16$

Ruenwai R, Neiss A, Laoteng K, Vongsangnak W, Dalfard AB, Cheevadhanarak S, Petranovic D, Nielsen J (2015) Heterologous production of polyunsaturated fatty acids in Saccharomyces cerevisiae causes a global transcriptional response resulting in reduced proteasomal activity and increased oxidative stress. Biotechnol J 6:343-356

Sahin D, Tas E, Altindag UH (2018) Enhancement of docosahexaenoic acid (DHA) production from Schizochytrium sp. S31 using different growth medium conditions. AMB Express 8:7 
Saygideger SD, Okkay O (2008) Effect of 2,4-dichlorophenoxyacetic acid on growth, protein and chlorophyll-a content of Chlorella vulgaris and Spirulina platensis cells. J Environ Biol 29:175-178

Song X, Tan Y, Liu Y, Zhang J, Liu G, Feng Y, Cui Q (2013) Different impacts of short-chain fatty acids on saturated and polyunsaturated fatty acid biosynthesis in Aurantiochytrium sp. SD116. J Agric Food Chem 61:9876-9881

Su CH, Liangjung C, Gomes J, Lin YS, Yu YK, Jhangsong L, Rongjhih S (2011) Factors affecting lipid accumulation by Nannochloropsis oculata in a twostage cultivation process. J Appl Phycol 23:903-908

Sun XM, Ren LJ, Bi ZQ, Ji XJ, Zhao QY, Jiang L, Huang H (2018) Development of a cooperative two-factor adaptive-evolution method to enhance lipid production and prevent lipid peroxidation in Schizochytrium sp. Biotechnol Biofuels 11:65

Tan KW, Lee YK (2016) The dilemma for lipid productivity in green microalgae: importance of substrate provision in improving oil yield without sacrific ing growth. Biotechnol Biofuels 9:255

Tian QL, Shi DJ, Jia XH, Mi HL, Huang XW, He PM (2014) Recombinant expression and functional analysis of a Chlamydomonas reinhardtii bacterialtype phosphoenolpyruvate carboxylase gene fragment. Biotechnol Lett 36:821-827

Trentacoste EM, Shrestha RP, Smith SR, Glé C, Hartmann AC, Hildebrand M, Gerwick WH (2013) Metabolic engineering of lipid catabolism increases microalgal lipid accumulation without compromising growth. Proc Natl Acad Sci USA 110:19748-19753

Xue J, Balamurugan S, Li DW, Liu YH, Zeng H, Wang L, Yang WD, Liu JS, Li HY (2017) Glucose-6-phosphate dehydrogenase as a target for highly efficient fatty acid biosynthesis in microalgae by enhancing NADPH supply. Metab Eng 41:212-221

Yu XJ, Sun J, Sun YQ, Zheng JY, Wang Z (2016) Metabolomics analysis of phytohormone gibberellin improving lipid and DHA accumulation in Aurantiochytrium sp. Biochem Eng J 112:258-268

Zhang J, Hao H, Chen M, Wang H, Feng Z, Chen H (2017) Hydrogen-rich water alleviates the toxicities of different stresses to mycelial growth in Hypsizygus marmoreus. AMB Express 7:107

Zhang S, He Y, Sen B, Chen X, Xie Y, Keasling JD, Wang G (2018) Alleviation of reactive oxygen species enhances PUFA accumulation in Schizochytrium sp. through regulating genes involved in lipid metabolism. Metab Eng Commun 6:39-48

Zhao Y, Shang M, Xu JW, Zhao P, Li T, Yu X (2015) Enhanced astaxanthin production from a novel strain of Haematococcus pluvialis using fulvic acid. Process Biochem 50:2072-2077

Zhao B, Li Y, Li C, Yang H, Wang W (2018) Enhancement of Schizochytrium DHA synthesis by plasma mutagenesis aided with malonic acid and zeocin screening. Appl Microbiol Biotechnol 102:2351-2361

\section{Submit your manuscript to a SpringerOpen ${ }^{\odot}$ journal and benefit from:}

- Convenient online submission

- Rigorous peer review

- Open access: articles freely available online

- High visibility within the field

- Retaining the copyright to your article

Submit your next manuscript at $\boldsymbol{\nabla}$ springeropen.com 